Article

\title{
Cavity Ring-Down Spectroscopy for Molecular Trace Gas Detection Using A Pulsed DFB QCL Emitting at $6.8 \mu \mathrm{m}$
}

\author{
Komlan S. Gadedjisso-Tossou 1,2,3(1), Lyubomir I. Stoychev ${ }^{3,4}{ }^{(0)}$, Messanh A. Mohou ${ }^{2}$, \\ Humberto Cabrera ${ }^{1,3, * \mathbb{D}}$, Joseph Niemela ${ }^{1}$, Miltcho B. Danailov ${ }^{5}$ and Andrea Vacchi ${ }^{3,6}$ \\ 1 Optics Lab, The Abdus Salam International Centre for Theoretical Physics, 34151 Trieste, Italy; \\ kgadedjisso@univ-lome.tg (K.S.G.-T.); niemela@ictp.it (J.N.) \\ 2 Laboratoire de Physique des Composants à Semi-conducteurs (LPCS), Département de Physique, Université \\ de Lomé, 01 BP 1515 Lomé, Togo; mmohou@univ-lome.tg \\ 3 Sezione di Trieste, Istituto Nazionale di Fisica Nucleare, 34149 Trieste, Italy; \\ Lyubomir.Stoychev@ts.infn.it (L.I.S.); vacchi@ts.infn.it (A.V.) \\ 4 Institute of Solid State Physics, Bulgarian Academy of Sciences, 72 Tzarigradsko Chaussee, \\ Sofia 1784, Bulgaria \\ 5 ELETTRA—Sincrotrone Trieste, Area Science Park, 34149 Trieste, Italy; miltcho.danailov@elettra.eu \\ 6 Department of Mathematics, Computer Science and Physics, University of Udine-Via Delle Scienze, 206, \\ 33100 Udine, Italy \\ * Correspondence: hcabrera@ictp.it
}

Received: 12 August 2020; Accepted: 9 September 2020; Published: 14 September 2020

\begin{abstract}
A trace gas sensor based on pulsed cavity ring-down spectroscopy (CRDS) was developed for measurement of the $v 4$ fundamental vibrational band of ammonia $\left(\mathrm{NH}_{3}\right)$ centered at $1468.898 \mathrm{~cm}^{-1}$. A pulsed distributed feedback quantum cascade laser (DFB-QCL) operating at $6.8 \mu \mathrm{m}\left(1470.58 \mathrm{~cm}^{-1}\right)$ quite well covered the absorption band of the ammonia and strong fundamental vibrational absorption bands of different molecular gases in this unexplored region. The cavity was partially evacuated down to $0.4 \mathrm{Atm}$ by a turbo-molecular pump to reduce the partial interference between the $\mathrm{NH}_{3}$ spectra and water near the absorption peak of ammonia. A sensitivity of nine parts per billion was reached for a measurement time of $120 \mathrm{~s}$ as well as an optical path length of $226 \mathrm{~m}$. The device demonstrated high spectral performance and versatility due to its wide tuning range, narrow linewidth, and comparatively high-energy mid-IR radiation in the relatively unexplored $6.8 \mu \mathrm{m}$ region, which is very important for high-resolution spectroscopy of a variety of gases.
\end{abstract}

Keywords: cavity ring-down spectroscopy; quantum cascade laser; mid-infrared spectral region; ammonia

\section{Introduction}

Mid-IR (MIR) absorption spectroscopy is an extremely effective tool for detection of molecular trace gases [1-3]. It is well appreciated that the MIR spectral wavelength range stretching from $3 \mu \mathrm{m}$ to $10 \mu \mathrm{m}$ contains a wealth of strong fundamental vibrational absorption bands of different molecular gases, and therefore is suitable for trace gas sensing applications, such as environmental monitoring, biomedical diagnostics, and control of toxic industrial chemicals [4]. For example, the detection of gases, such as $\mathrm{CH}_{4}, \mathrm{CO}_{2}, \mathrm{NO}_{2}, \mathrm{SO}_{2}$, and $\mathrm{NH}_{3}$, at the level of parts per billion (ppb) plays an essential role in environmental and agricultural monitoring.

Ammonia is the third most abundant nitrogen-containing compound in the atmosphere, playing an essential role in the nitrogen cycle [5]. It is mostly used in agricultural activities to manufacture 
fertilizers such as ammonium phosphate, ammonium nitrate, and ammonium sulphate. Ammonia is also used for the synthesis of many pharmaceuticals and commercial cleaning products. Besides pollution effects, ammonia can cause health hazards with brief exposure to concentrations greater than 1500 parts per million (ppm) [6]. The typical concentration of ammonia in atmosphere or in human exhaled breath is in the range from parts per billion to part per trillion by volume. Therefore, there is a need for sensitive detection of ammonia in the atmosphere and monitoring its levels for environmental and medical purposes. In the MIR region, the earliest and recent investigations on $\mathrm{NH}_{3}$ were mostly focused in the study of the rotational-vibrational transition bands in the wavelength range of $1.5 \mu \mathrm{m}$ to $3 \mu \mathrm{m}$ by using high-resolution spectroscopic analysis techniques [7-12]. More recently, the highly sensitive detection of part per billion of the $v 2$ fundamental vibrational band of $\mathrm{NH}_{3}$ around $10.3 \mu \mathrm{m}$ was reported [13-17]. Although the absorption lines of $\mathrm{NH}_{3}$ are very strong in the wavelength region centered at $10 \mu \mathrm{m}$, this region is affected substantially by the interference of $\mathrm{CO}_{2}$ absorption lines. Therefore, there is a need to explore another wavelength region over which the absorption lines of $\mathrm{NH}_{3}$ present stronger absorption than in the $10 \mu \mathrm{m}$ region. Interestingly, the region centered at $6.6 \mu \mathrm{m}$ has strong absorption lines which are additionally free of the interference of $\mathrm{CO}_{2}$. Unfortunately, in this region, water absorbs strongly, making the region less explored. Only Maithani et al. reported, in 2018, high resolution and high-sensitive spectroscopic analysis of $\mathrm{NH}_{3}$ centered at $6.2 \mu \mathrm{m}$ [18]. Therefore, further work is needed in the wavelength ranges between 5 and $7 \mu \mathrm{m}$ where strong absorption lines of fundamental and combinational vibrational bands of $v 4$ and $v 2$ are located. Exploring these absorption bands allows alternative measurements as well as spectroscopic studies with excellent performance and higher sensitivity $[19,20]$. Furthermore, this wavelength region is suitable in spectroscopic applications in medicine and biology for the detection of proteins [21,22]. Moreover, the spectral region around $6.8 \mu \mathrm{m}$ is very important for the high-precision spectroscopic measurement of the hyperfine structure of muonic hydrogen and contains the footprints of several important unsaturated hydrocarbons such as isoprene [23-25].

Many techniques based on direct and indirect absorption spectroscopy of atoms and molecules in gas phase have been developed in order to achieve a sensitive and accurate measurement of trace gases [26-28]. Among direct absorption techniques, the cavity ring-down spectroscopy (CRDS) technique has proven high sensitivity and accuracy due to its independence of laser noise [29-34]. The CRDS differs from the other techniques because it uses a time-based measurement to perform the absorption spectrum of the gas, rather than traditional intensity-based absorption techniques. In CRDS, the light from a laser source is confined in a high-finesse optical cavity in order to increase the effective absorption path length significantly. Moreover, beyond the detection technique, tunable and high power light sources operating in MIR wavelength region are needed in order to have high selectivity as well as high-sensitivity for precise and quantitative analysis. Quantum cascade lasers (QCLs) are the ideal sources for gas sensing in this wavelength region. QCLs are unipolar intersubband lasers in which the radiative transitions occur in one band and involve a single type of carrier, namely electrons. The first QCL was demonstrate in 1994 by Faist and al [35]. Research in this field is continuously expanding and gives today, laser sources covering the entire mid-infrared and terahertz spectral region for sensitive detection of molecular species. QCLs offer high power, high spectral performance, and a reliable, coherent light source. A stringent single-mode emission and superior wavelength stability as required for trace gas sensing are met by distributed feedback (DFB)-QCLs [36-39].

The goal of this work was to build a high-resolution pulsed-CRDS sensor to measure molecular trace gases, first using ammonia as a test sample in a well-controlled environment and exploring the spectral region with wavelengths near $6.8 \mu \mathrm{m}$. The sensor is based on a pulsed DFB-QCL operating around $6.8 \mu \mathrm{m}$ which match well with the $v 4$ fundamental vibrational band of ammonia centered at $1468.898 \mathrm{~cm}^{-1}$. In order to reduce the partial interference of $\mathrm{NH}_{3}$ and $\mathrm{H}_{2} \mathrm{O}$ lines in this wavelength range, the CRDS, which is based on a 50-cm-long optical cavity, was preliminarily evacuated by a turbo molecular pump. A part per billion level of detection sensitivity was reached using an optical path length of $226 \mathrm{~m}$. 
The design of this system, with its tunability and high spectral performance, allows a straightforward extension to important remote gas sensing applications involving other gas molecules with absorption bands falling within the same interval.

\section{Theory}

The CRDS technique was first introduced in 1988 [29] and is based on measurement of the decay rate of light trapped and bouncing repeatedly inside a high finesse optical cavity. When a laser pulse is transmitted into the cavity, it will gradually leak out of the cavity at each reflection through the highly reflecting mirrors. The intensity of the light transmitted through the output mirror decays exponentially with a time constant as [27]:

$$
I(t)=I_{0} \exp \left(-\frac{t}{\tau}\right)
$$

where $I_{0}$ is the initial detected light intensity and $\tau$ is the time constant named ring-down time. The ring-down time is the time for which the intensity reaches $1 / \mathrm{e}$ of $I_{0}$.

The general ring-down time $\tau$ is then given by:

$$
\tau(v)=\frac{L}{c \cdot\left[(1-R)+\sum_{i} \sigma_{i}(v) \int_{0}^{L} N_{i}(x) \mathrm{d} x\right]}
$$

where $L$ is the distance between the mirrors, $R$ is the mirror reflectivity, $c$ is the speed of light, $\sigma_{i}(v)$ is the frequency-dependent absorption or scattering cross-section for each transition of each species, $\int_{0}^{L} N_{i}(x) \mathrm{d} x$ is the line-integrated number density, and the sum is over all species inside the cavity.

In an empty cavity, the sum term vanished and the ring-down time $\tau_{0}$ depends only on the mirror reflectivity, the distance between the mirrors and the speed of the light.

$$
\tau_{0}=\frac{L}{c \cdot(1-R)}
$$

For the gas phase samples, the scattering can be neglected as compared to the absorption. Moreover, if the gas species are homogeneous, the ring-down time can be written as follow.

$$
\tau(v)=\frac{L}{c \cdot[(1-R)+\alpha(v) \cdot L]}
$$

where $\alpha(v)$ is the absorption coefficient of species at frequency $v$.

The absorption coefficient of the sample present in the cavity is obtained by fitting Equations (3) and (4).

$$
\alpha(v)=\frac{1}{c}\left(\frac{1}{\tau(v)}-\frac{1}{\tau_{0}}\right)
$$

The sensitivity of the CRDS technique depends on the measurement precision of the ring-down time and is given by:

$$
[\alpha(v)]_{\min }=\sigma(v)[N]_{\min }=\frac{1}{c \tau_{0}^{2}} \Delta \tau_{\min }
$$

where $\Delta \tau_{\min }$ is the standard deviation of the empty cavity ring-down time, $\sigma(v)$ is the absorption cross-section at the absorption wavelength, and $N$ is the number density of the molecule.

\section{Experimental Setup}

The schematic representation of the experimental set-up is shown in Figure 1. The cryogenic DFB-QCL is mounted on a sample holder with a liquid nitrogen-cooled cryostat to achieve the required 
temperature range. The cryostat (OptistatDN-V, Oxford Instruments) is controlled by an external PID temperature controller (ITC503, Oxford Instruments). Therefore, it is possible to stabilize the temperature of the probe mount very accurately $(0.1 \mathrm{~K})$ in the range of $77-500 \mathrm{~K}$. In order to operate the cryostat properly, good vacuum conditions $\left(10^{-4} \mathrm{mbar}\right)$ are necessary to avoid thermal dissipation, vapor condensation and freezing of the bath's outer chamber. A turbomolecular pump backed by a membrane pump (ILMVAC CD160) is used to achieve proper vacuum conditions. This pump can create a vacuum up to $10^{-7}$ mbar. The laser was operated in a pulsed mode and driven by a pulser (LDD-TPG 228, Alpes Lasers) with pulse widths ranging from $50 \mathrm{~ns}$ to $200 \mathrm{~ns}$ and laser drive current from 1 to $2.3 \mathrm{~A}$.

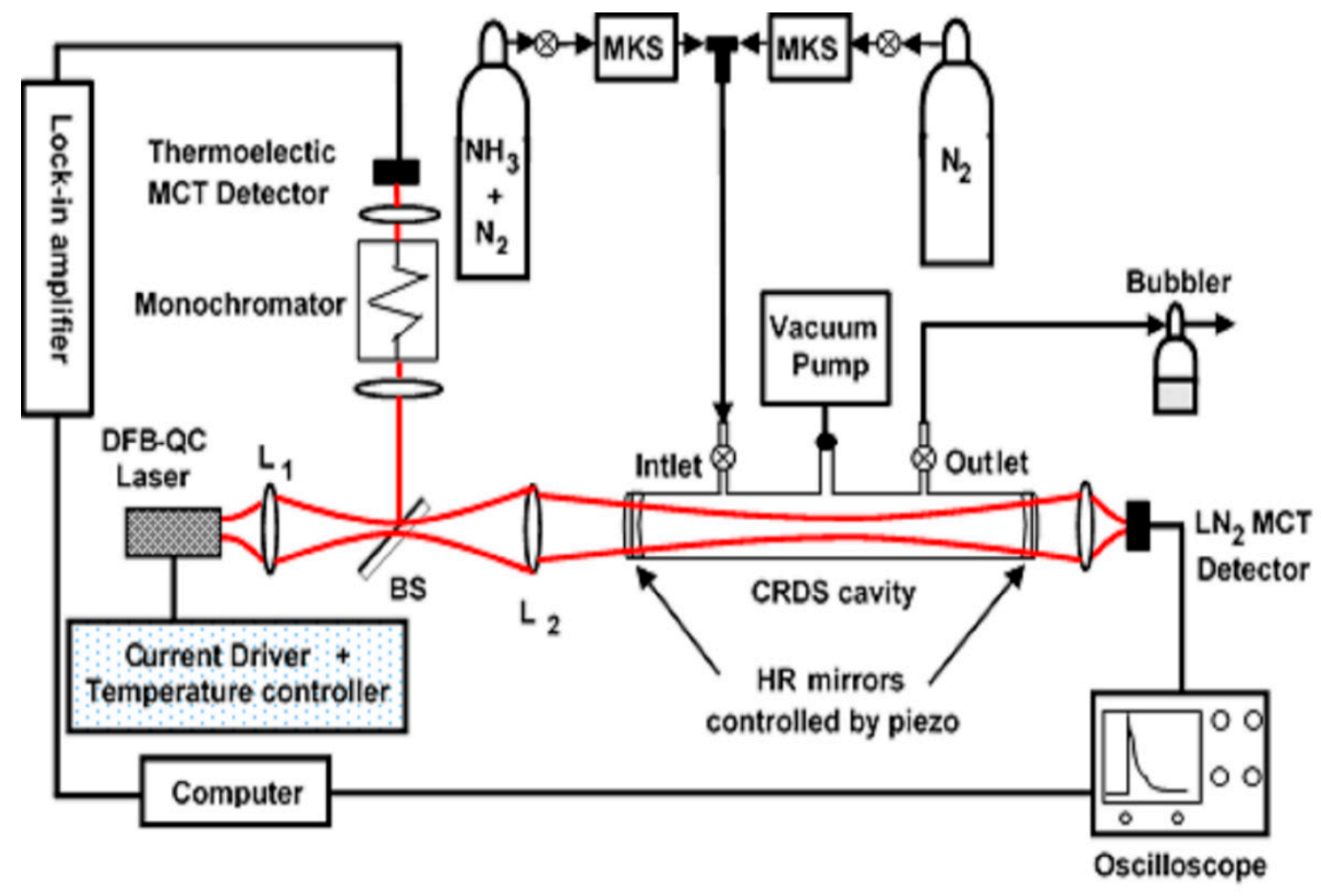

Figure 1. Schematic representation of the cavity ring-down spectrometer setup.

The optical cavity is part of a quartz-coated vacuum chamber and consists in two identical plano-concave mirrors of $1 \mathrm{~m}$ radius of curvature and $25.40 \mathrm{~mm}$ diameter separated by $50 \mathrm{~cm}$ $(d=50 \mathrm{~cm})$ apart (Los Gatos Research, Inc., Mountain View, CA, USA). The reflection coefficient of the mirrors at $6.80 \mu \mathrm{m}$ is $99.8 \%$ (II-VI INFRARED, $\mathrm{R}=99.8 \%$ at $6.8 \mu \mathrm{m}$ ). The mirrors are made of a ZnSe substrate, with anti-reflection coatings on the plane sides and dielectric coatings on the concave surfaces. Each mirror can be adjusted by using an assembly of three piezoelectric actuators (15 microns of piezo-electric travel from 0-150 V, MDT693A Piezo Controller).

A pulsed DFB-QC laser emitting around $6.80 \mu \mathrm{m}$ (Alpes Laser, \#sb 1486 DN) was used. The DFB-QCL was operated at a repetition rate of $200 \mathrm{kHz}$ and $100 \mathrm{~ns}$ pulse width (2\% duty cycle), allowing us to scan the wavenumber range from 1465.11 to $1474.88 \mathrm{~cm}^{-1}$ by changing the operating temperature from $80 \mathrm{~K}$ to $180 \mathrm{~K}$. The maximum average output power of $20 \mathrm{~mW}$ was achieved at $2 \%$ duty cycle. For the mode-matching between the laser beam and the cavity mode, we have used antireflection-coated lenses of $\mathrm{Ge}\left(\mathrm{L}_{1}\right)$ and $\mathrm{CaF} 2\left(\mathrm{~L}_{2}\right)$ with focal lengths of $25.4 \mathrm{~mm}$ and $200 \mathrm{~mm}$, respectively. Between the two lenses, the laser beam is divided into two parts by a beamsplitter (BS). One part of the beam goes directly into the CRDS cavity and the second part to the monochromator (MS257, MODEL 77700A, SPECTRA PHYSICS) for the real-time measurement of the wavelength. The output signal from the monochromator is focused into a thermoelectric HgCdTe photovoltaic detector (VIGO PVMI-10.6) which is connected to a lock-in amplifier (SIGNAL RECOVERY 7280 DSP) and a 2 GHz oscilloscope (WaveRunner 6200A, LeCroy). The lock-in amplifier is triggered with the 
QC-laser trigger and is used for extracting the signal from the VIGO detector. The output light of the cavity is focused by a Ge plano-convex lens of $25.40-\mathrm{mm}$-diameter and $25.40 \mathrm{~mm}$ focal length into a liquid-nitrogen-cooled $\mathrm{HgCdTe}$ photovoltaic detector (Cryogenic HgCdTe detector, Kolmar-model KMPV11-0.5-J2, $20 \mathrm{MHz}$ bandwidth, sensing area of $0.5 \times 0.5 \mathrm{~mm}$ ). The output signal from the detector is connected to a LeCroy WaveRunner 6200 A digitizing oscilloscope (5 GS/s sample rate, $2 \mathrm{GHz}$ bandwidth and 8 bits vertical resolution) and a Gage CompuScope high speed digitizer card (GaGe CS 12100, 12 bit, $100 \mathrm{MS} / \mathrm{s}$ ) mounted on a standard computer. The exponential decay signal was captured with Gage card and processed using LGR's proprietary pre-processor and (least-squares) fitting algorithms wrapping in LabVIEW software. The alignment of the whole optical system and the calibration of the monochromator were performed using a green laser operating at $532 \mathrm{~nm}$ wavelength.

The initial part of our work consisted in the optimization of the mode matching between the laser beam and the optical cavity. To facilitate the longitudinal mode matching, we first performed a simulation based on the ray-transfer matrix to find the best positions of the lenses and cavity mirrors that could allow us to match the beam waist to the cavity waist at the middle of the cavity.

Subsequently, the end mirror of the cavity was approximately placed at the calculated position from the front of the QCL laser. A green laser passing through a pinhole located in the place of the first mirror was used to adjust the mirror. The PZT actuators of the end mirror were adjusted to ensure the overlapping of the feedback light from the mirror with the pinhole aperture. Then, the lenses $L_{1}$ and $\mathrm{L}_{2}$ were placed at the calculated positions. Thereafter, the PZT actuators of the front mirror of the cavity were adjusted roughly by hand to have a smooth single-exponential CRDS decay and the PZT actuator controllers (from the center voltage of the controller, $75 \mathrm{~V}$ ) of $\mathrm{L}_{2}$ and the two mirrors were activated. The voltage of controllers was adjusted to have the smoothest decay and to improve the value of the initial intensity of the CRDS decay, which is expected to guarantee also the best longitudinal mode matching. It was estimated that the drifts on resonant cavity mode are negligible during our measurement time; in fact, during the measurement time (after each mode matching setting) typical temperature variations of less than 1 degree Celsius were observed. Taking into account that the thermal expansion coefficient of the quartz-coated cavity is in the order of $10^{-6} \mathrm{~K}^{-1}$, and that cavity mode spacing of the cavity (FSR) is about $300 \mathrm{MHz}$, the corresponding frequency shift of the cavity mode, due to a 1-degree temperature change, would be less than $200 \mathrm{~Hz}$, i.e., much smaller compared to the theoretical cavity linewidth $\left(\gamma=1 / 2 \pi \tau_{0}\right)$, which is $191 \mathrm{kHz}$.

\section{Results}

The stability of the optical cavity was analyzed by means of an Allan variance test to find the optimized time for averaging the measurement. For this purpose, decay signals were recorded for different amounts of signal averaging, from 1, 2, 4, 8, 16, 32, 64, 128, 256, 512, 1024, and 2048 averaged decays. Figure 2 shows the Allan variance of the empty cavity ringdown $\left(\tau_{0}\right)$ as function of the number of the averaged decays [26]. The log-log plot of the Allan variance shows that the Allan variation decreases with increasing averaging (due to the fact that noise decreases with more data averaging) up to an optimum value of 256 averaging decays, then increases due to temperature changes, electronic components effects, or other possible factors. From Figure 2, we infer that the optimal sensitivity occurs with the averaging of 256 decays. Figure 3 shows the ring-down decay recorded with 256 shots averaging at the wavenumber of $1470.58 \mathrm{~cm}^{-1}(6.80 \mu \mathrm{m})$ for the evacuated cavity at a pressure around $10^{-7}$ mbar (black color). The portion before the surge part of the signal is the baseline signal of the detector and is used to determine the offset. The surge part corresponds to the laser pulse injection into the CRDS cavity. The decay curve is used to calculate the ringdown time $\tau$. The ringdown time was calculated by fitting the experimental data to an exponential decay function $A \exp (-t / \tau)+B$, where $A$ is the initial intensity of the light and $B$ is the DC offset of the detector. A value of $\tau_{0}=754.30 \mathrm{~ns}$ was calculated corresponding to an effective mirror reflectivity of $99.77 \%$ and an effective optical path length $d /(1-R)$ of $226 \mathrm{~m}$. This experimental value of the reflection coefficient fits well with the reflection coefficient value range given by the supplier, $R=99.8 \%( \pm 0.15 \%)$ at $6.8 \mu \mathrm{m}$. Based on this 
ringdown time value the cavity linewidth $\left(\Delta \gamma=1 / 2 \pi \tau_{0}\right)$ was estimated to be $211 \mathrm{kHz}$ and the finesse of the optical cavity $(F=F S R / \Delta \gamma)$ to be 1421 .

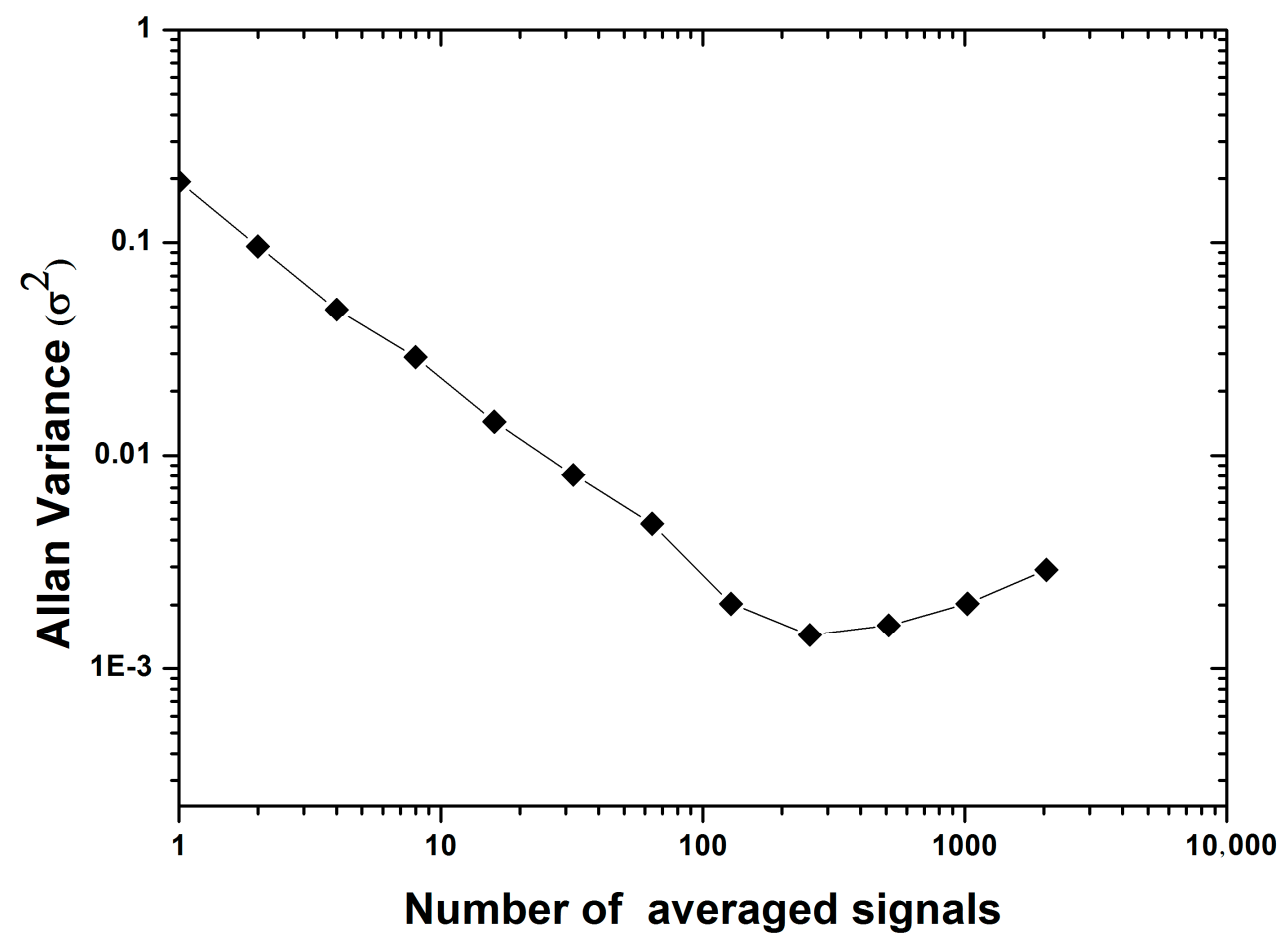

Figure 2. Allan Variance plot of empty pumped cavity ringdown time as function of the average decays.

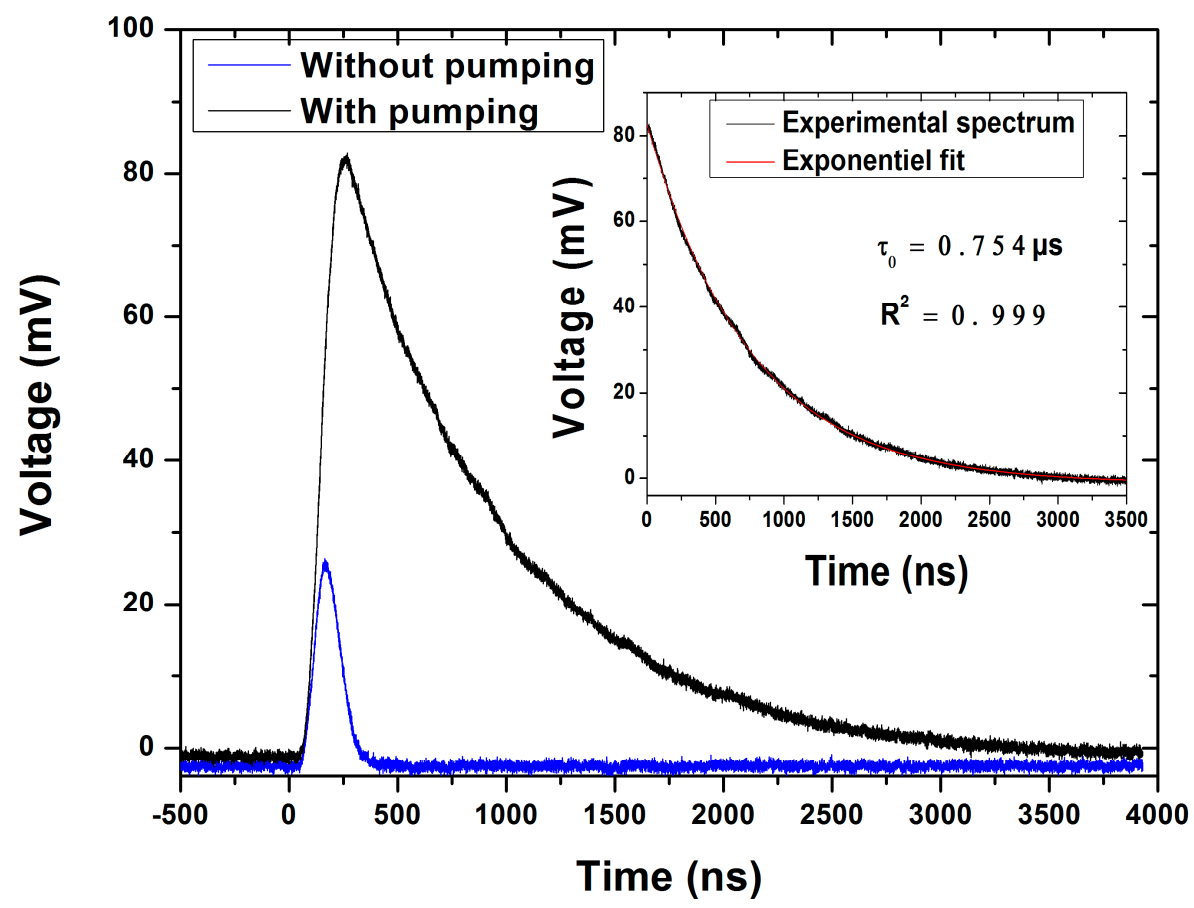

Figure 3. Ring-down decay recorded at a wavelength of $6.80 \mu \mathrm{m}$ for the pumped empty cavity composed of a 50-cm-long optical cavity and mirrors with reflectivity of $99.77 \%$ (black) and a cavity with an atmospheric pressure of air (blue). The inset shows the fitted exponential decay curve of the pumped empty cavity. 
Figure 4a shows the simulated spectral lines obtained from HITRAN database of $\mathrm{NH}_{3}$ in the MIR spectral region covering $500.00-3500.00 \mathrm{~cm}^{-1}$ [4]. The $v 4$ fundamental vibrational band of $\mathrm{NH}_{3}$ centered at $1500 \mathrm{~cm}^{-1}(6.6 \mu \mathrm{m})$ shows intense lines but remains less attractive due to the strong absorption of water $\left(\mathrm{H}_{2} \mathrm{O}\right)$ in this region (Figure $\left.4 \mathrm{~b}\right)$. However, this region has advantage for gas analyses because is free from $\mathrm{CO}_{2}$ interference. The absorption feature centered on $1468.898 \mathrm{~cm}^{-1}$ was chosen for our studies. Figure $4 \mathrm{c}$ shows the simulation of the absorption spectra of $\mathrm{NH}_{3}(23 \mathrm{ppm})$ and $\mathrm{H}_{2} \mathrm{O}(5 \%)$ for an optical pathlength of $226 \mathrm{~m}$ and a temperature of $296 \mathrm{~K}$ for three different pressures, i.e., 1, 0.4, and $0.1 \mathrm{Atm}$, in the wavenumber range between $1460 \mathrm{~cm}^{-1}$ and $1476 \mathrm{~cm}^{-1}$. It is worth observing in Figure $4 \mathrm{c}$ that the pressure induced broadening of the spectral line. The challenge is to operate the gas sensor setup at a pressure closer to $1 \mathrm{Atm}$ in order to use this device in normal atmospheric condition for spectroscopy. From the figure, it is clear that the overlapping of the bands is pressure dependent and that, reducing the pressure, the $\mathrm{NH}_{3}$ and $\mathrm{H}_{2} \mathrm{O}$ lines will reduce the level of overlapping due to the decreasing of the baseline of the $\mathrm{H}_{2} \mathrm{O}$ absorption. A pressure of $0.4 \mathrm{Atm}$ was chosen for our work in order to reduce the water absorption baseline as well as the broadening of the ammonia spectrum. To reduce the partial interference between $\mathrm{NH}_{3}$ and $\mathrm{H}_{2} \mathrm{O}$ at this pressure, a turbo molecular pump backed by a membrane pump (ILMVAC CD160) was used to pump the optical cavity to reach a proper vacuum prior to measurement. After that a constant flow of nitrogen $\left(\mathrm{N}_{2}\right)$, was used to additionally purge, was left through the cavity. Finally, a constant flow of nitrogen-ammonia mixture at a specific concentration was generated using a gas dilution system. First, an ammonia gas 100 ppmvol with 1.9 ppmvol uncertainty (produced by SIAD SpA) was diluted with nitrogen at a pressure of $0.4 \mathrm{Atm}$. Needle valves were used to control the ammonia and nitrogen flow to reach a given concentration. Two mass flow meters (MKS) were used to read the flow rates for these gases. Samples with different concentrations of ammonia flowed continuously through the cavity. For monitoring the gas flow, a bubbler was placed at the outlet of the cavity. A comparison between CRDS signal without pumping and the signal obtained after evacuating (Figure 3), showed a drastic improvement of the signal since the interference from water vanished. Decay times of $0.754 \mu$ s and $0.074 \mu$ s were calculated for the pumped and not pumped cavity, respectively.

In order to record the spectrum of ammonia, the QCL was tuned from $1467.163 \mathrm{~cm}^{-1}$ to $1470.395 \mathrm{~cm}^{-1}$ by changing the laser heat-sink temperature from $134 \mathrm{~K}$ to $166 \mathrm{~K}$ with a tuning rate of $-0.101 \mathrm{~cm}^{-1} \mathrm{~K}^{-1}$. Spectra of different concentration of ammonia 9, 17, 23, 28, and $33 \mathrm{ppm}$ were recorded. For each measurement, the cavity was emptied and then filled again with different concentrations of $\mathrm{NH}_{3}$ gas at the same pressure. The CRDS signal obtained with the pure $\mathrm{N}_{2}$ gas in the chamber was used as the blank signal. Figure 5 shows the CRD decays measured from the pumped cavity, the cavity filled with nitrogen, and a 9 ppm concentration of ammonia in the cavity. The spectra measured at various concentration of ammonia from 9 to 33 ppm are shown in Figure 6. The absorption coefficients were calculated from Equation (5). For the data-acquisition, 256 ringdown events were averaged for each measurement. After some testing, we found that the time taken for having a constant $\mathrm{NH}_{3}$ flow in the cavity is about $115 \mathrm{~s}$. So, the signal was averaged and recorded over the measurement time of $120 \mathrm{~s}$. The repeatability was verified by performing the measurement three times. The relative standard deviation of the decay time at different wavenumbers is in the range of $0.05 \%$ to $0.1 \%$. Figure 7 shows a comparison of $\mathrm{NH}_{3}$ spectrum obtained at $23 \mathrm{ppm}$ with a simulated spectrum from the HITRAN database under same experimental conditions, i.e., $0.4 \mathrm{Atm}$ pressure and $296 \mathrm{~K}$ temperature. 

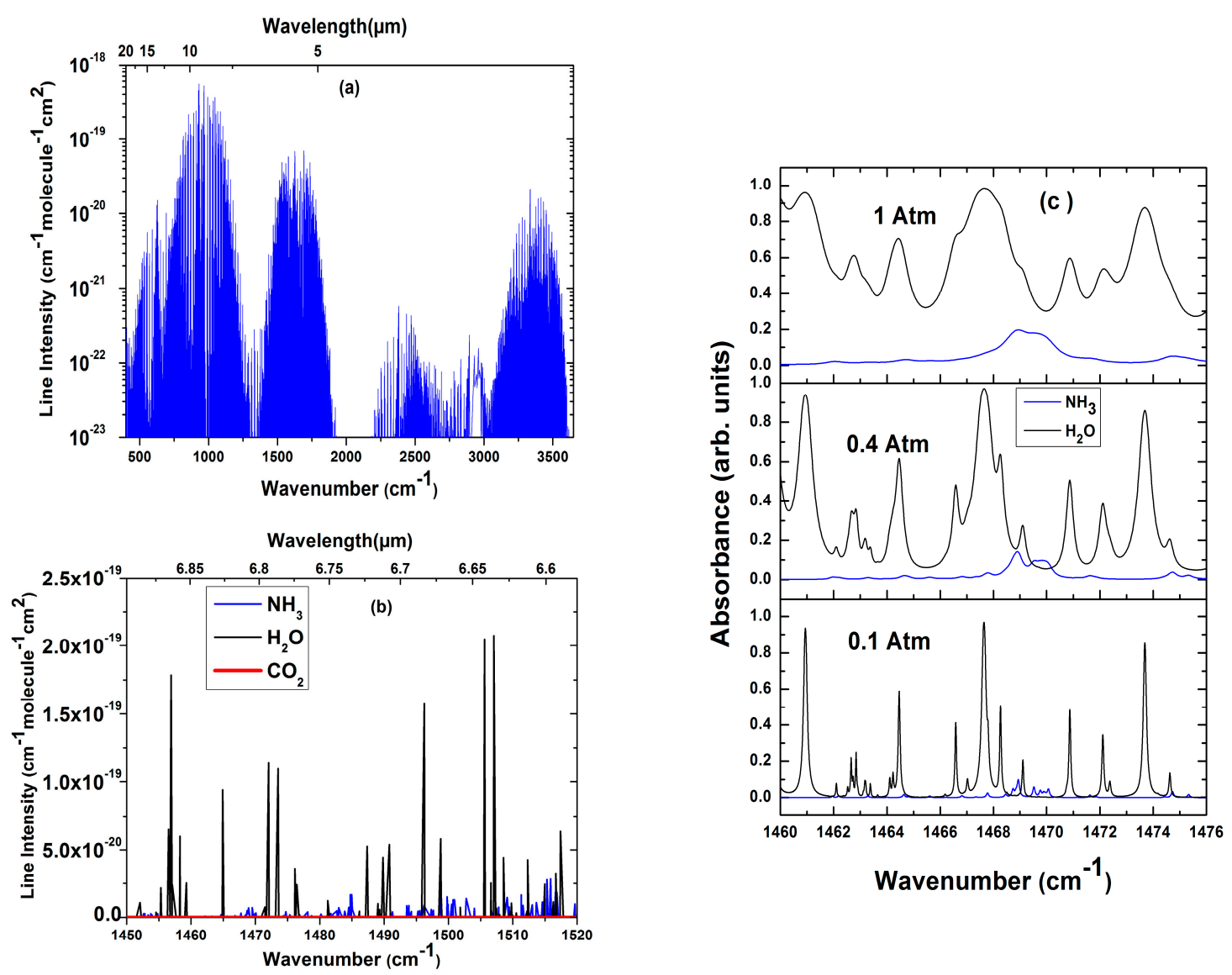

Figure 4. (a) HITRAN simulation lines intensities of $\mathrm{NH}_{3}$ in the wavelength range from $2.5 \mu \mathrm{m}$ to $25 \mu \mathrm{m}$, (b) HITRAN simulation line intensities of $\mathrm{NH}_{3}, \mathrm{CO}_{2}$ and $\mathrm{H}_{2} \mathrm{O}$ around $1470.58 \mathrm{~cm}^{-1}$, (c) HITRAN simulation of $\mathrm{NH}_{3}(23 \mathrm{ppm})$ spectra in the wavelength range of $6.80 \mu \mathrm{m}$ at a temperature of $296 \mathrm{~K}$ and pathlength of $226 \mathrm{~m}$ for three different pressures with $\mathrm{H}_{2} \mathrm{O}(5 \%)$ spectra.

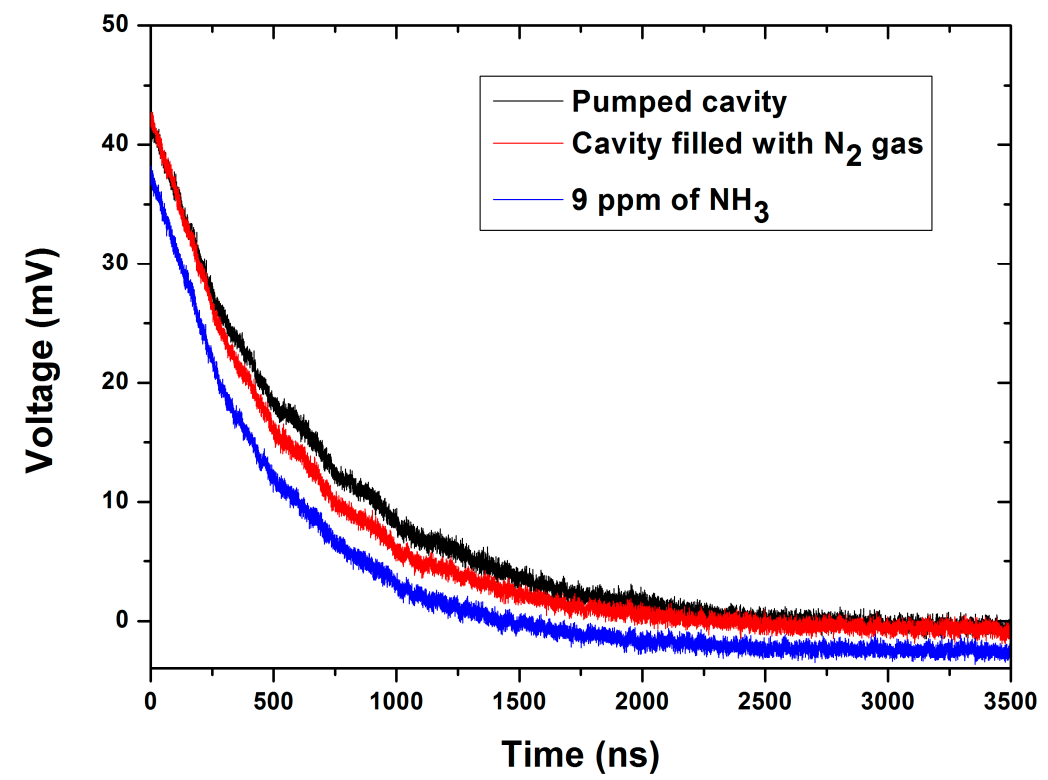

Figure 5. CRD decays recorded for the pumped, nitrogen filled and 9 ppm concentration of ammonia at wavenumber of $1469.852 \mathrm{~cm}^{-1}$. 


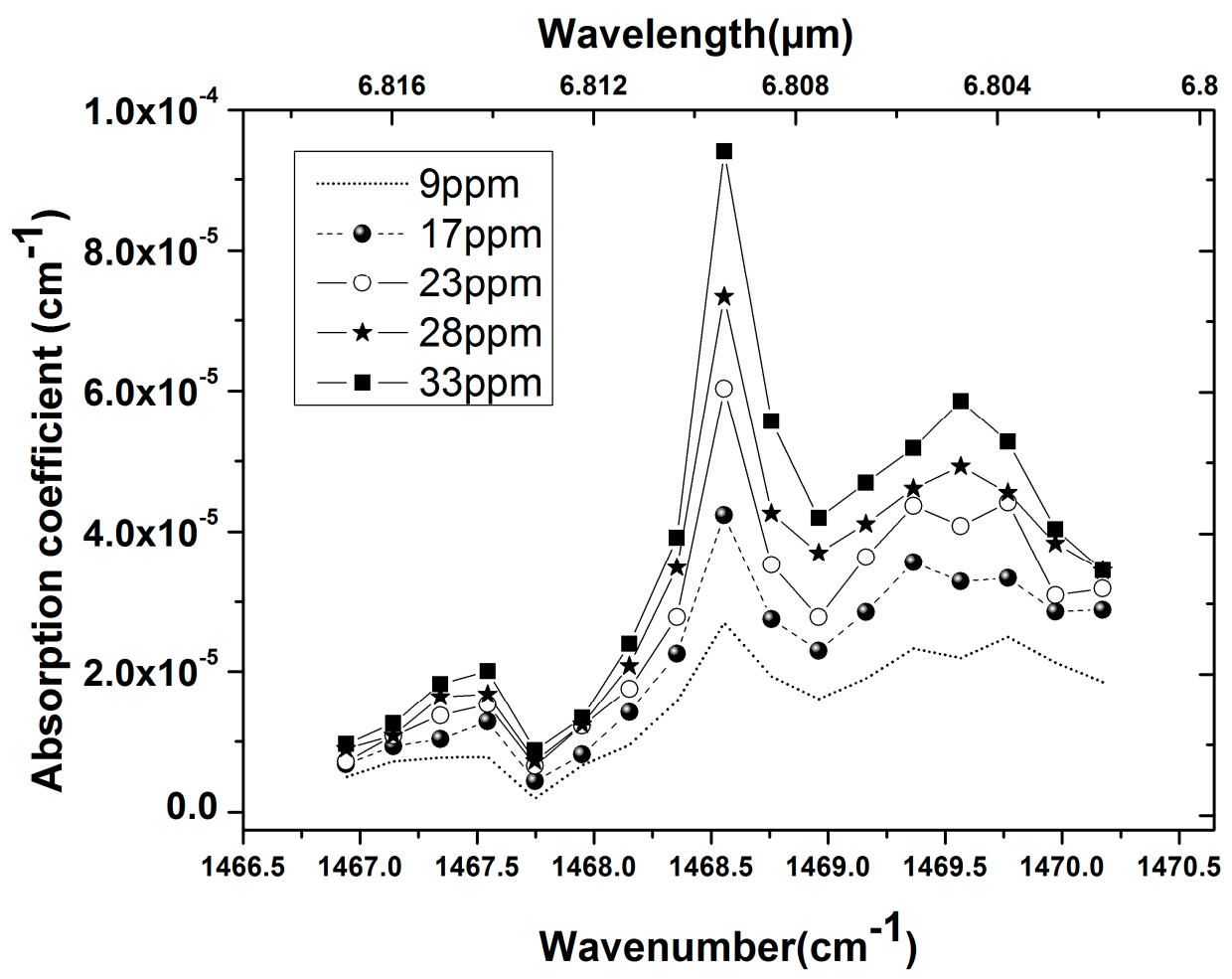

Figure 6. Absorption spectra of different concentrations of ammonia from 9 to $33 \mathrm{ppm}$ at a pressure of $0.4 \mathrm{Atm}$

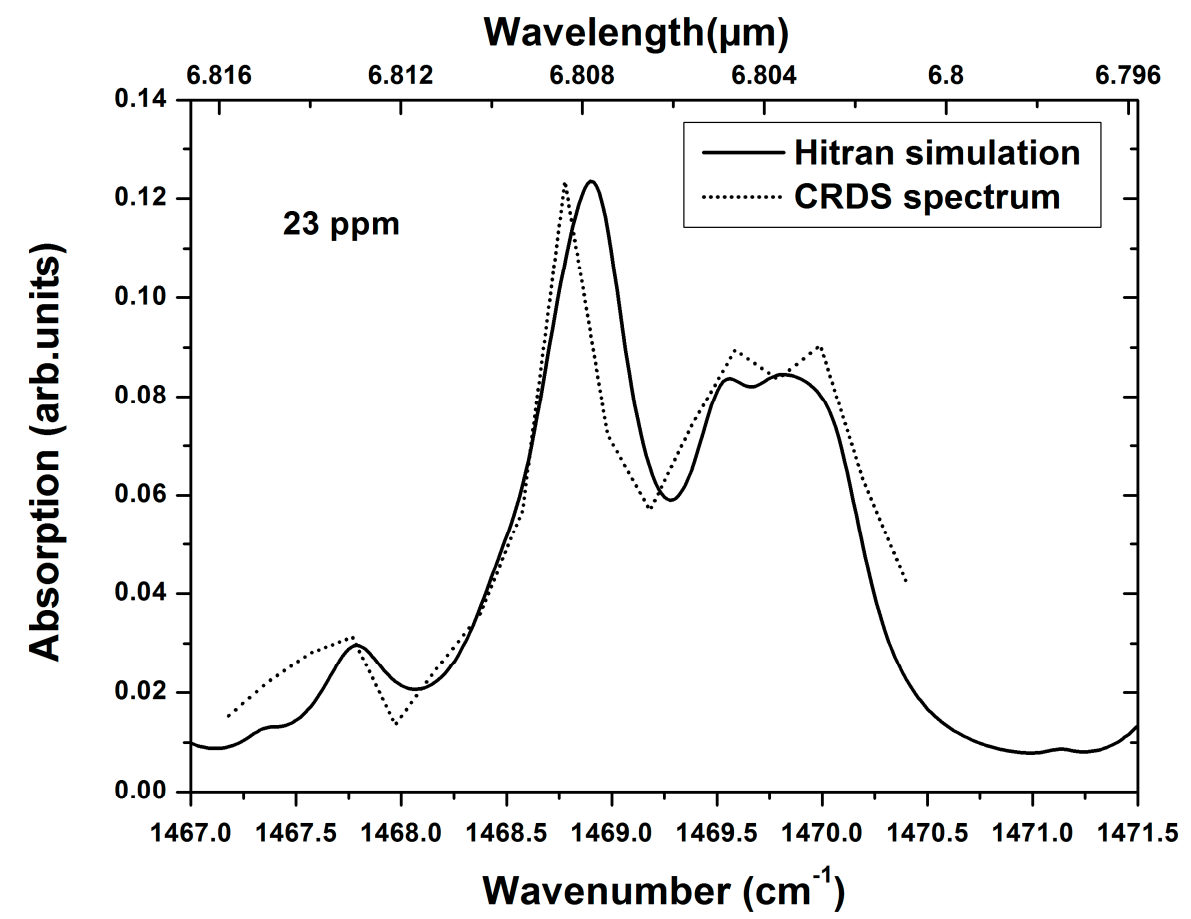

Figure 7. Measured absorption spectrum for $23 \mathrm{ppm}$ of ammonia at a pressure of 0.4 Atmosphere and a temperature of $296 \mathrm{~K}$ compared to the HITRAN simulation obtained under the same experimental conditions.

The limiting sensitivity of the setup was calculated from Equation (6) by $[N]_{\min }=\left(1 / c \sigma \tau_{0}\right) \Delta \tau_{\min } / \tau_{0}$. The absorption cross-section of ammonia at $1468.736 \mathrm{~cm}^{-1}$ wavenumber, is $2.3 \times 10^{-20} \mathrm{~cm}^{2}$ molecule ${ }^{-1}$. At this wavenumber, a decay time $\tau_{0}=754.3 \mathrm{~ns}$ with the relative 
standard deviation of $\Delta \tau_{\min } / \tau_{0}=0.005 \%$ was calculated corresponding to a minimum detectable absorption coefficient $\left(\alpha_{\min }\right)$ of $2.21 \times 10^{-8} \mathrm{~cm}^{-1}$. Using this value and ideal gas law, a detection limit of nine parts per billion by volume is obtained at room temperature and a pressure of 0.4 atmosphere.

\section{Discussion}

The results shown in Figure 7 indicate a good qualitative agreement between the two spectra, but there is a small shift around $0.1115 \mathrm{~cm}^{-1}$ of the wavenumber at peak $\left(1468.898 \mathrm{~cm}^{-1}\right)$ of the absorption line. This is probably due to the pressure induced wavelength shift because of high pressure.

The reported detection limit in this paper is somewhat higher (roughly ten times) than the earlier reported measurement in this wavelength range [18] but it is important to point out that those results were obtained at a very low pressure (1 Torr) and with a cw-CRDS setup with mirror reflectance greater than $99.98 \%$. By comparison, our results were obtained at a relatively high pressure of 304 Torr, using a pulsed-CRDS setup with mirror reflectance less than $99.80 \%$. In fact, obtaining a lower detection limit was not our primary goal because already $9 \mathrm{ppb}$ is enough to demonstrate the capabilities of our device. The difference in the detection limits is easily overcome by increasing the reflectivity of mirror's cavity; however, here wavelength selectivity is a more important advantage in order to tune the central emission at the maximum of absorption of different gases, thereby increasing the versatility of the device. We have demonstrated the capabilities of our setup to operate in an unexplored optical region where the absorbance of many gases take place without other interference effects. This was made possible due to a smooth tunability with short tuning step over a wider spectral region around $6.78 \mu \mathrm{m}$.

\section{Conclusions}

We have developed a cavity ring-down spectroscopic sensor for trace gas detection using a pulsed DFB QC laser emitting in the region around $6.8 \mu \mathrm{m}$. The system was demonstrated to be a high-resolution spectrometer able to analyze experimentally the absorption line of the $v 4$ fundamental vibrational band of ammonia centered at $1468.898 \mathrm{~cm}^{-1}(6.807 \mu \mathrm{m})$. A comparison between our CRDS measurements and the HITRAN database spectra shows good qualitative agreement. With the sensor employed, and a $50-\mathrm{cm}$-long optical cavity with mirrors of 0.998 reflectance, we were able to demonstrate the detection of ammonia with a sensitivity of $9 \mathrm{ppbv}$ and a $120 \mathrm{~s}$ measurement time. This test result shows that a pulsed laser-based-CRDS coupled with a cavity reflecting mirrors of $99.8 \%$ can be used as a highly sensitive chemical sensor.

In perspective, the sensitivity can be improved by performing detection using an optical cavity with higher reflectivity mirrors $(R>99.9)$ and lower gas concentrations. The work opens new possibilities in this unexplored spectral region because the high sensitivity detection of gases will be of substantial interest for several remote gas sensing applications in atmospheric chemistry, climate studies, as well as in biomedical research and diagnostics by measuring the release of relevant volatile compounds. Fundamental rovibronic absorption bands of a variety of gas molecules fall within this interval, such as water vapor, nitrous oxide, nitrogen oxide, nitrogen dioxide, methane, ethane, sulfur oxide, ammonia, hydrogen sulfide, nitric acid, and several others.

Author Contributions: Conceptualization, methodology, software, writing-original draft preparation, writing—review and editing, K.S.G.-T.; methodology, conceptualization, L.I.S.; writing—review and editing, M.A.M.; visualization, writing - review and editing, funding, H.C.; funding, review and editing, J.N.; review and editing, M.B.D.; project administration, funding acquisition, supervision, writing—review and editing, A.V. All authors have read and agreed to the published version of the manuscript.

Funding: This research received no external funding.

Acknowledgments: We gratefully acknowledge the financial support from the Abdus Salam International Centre for Theoretical Physics (ICTP-IAEA) through the TRIL programme, INFN (Sezione di Trieste) for provision of research facilities and in particular the SPIE-ICTP Anchor Research Program funded generously by the International Society for Optics and Photonics (SPIE). We express our gratitude to all members of the ICTP-Mlab and INFN 
Sezione di Trieste. We wish to thank Dr. Stefano Levorato, INFN Sezione di Trieste, for his technical help during this work.

Conflicts of Interest: The authors declare no conflict of interest.

\section{References}

1. Sigrist, M.W.; Kolthoff, I.M. Air Monitoring by Spectroscopic Techniques; Wiley: New York, NY, USA, 1994.

2. Meyers, R.A. Encyclopedia of Analytical Chemistry; Wiley: New York, NY, USA, 2000.

3. Sorokina, I.T.; Vodopyanov, K.L. Solid-State Mid-Infrared Laser Sources; Springer: Berlin, Germany, 2003.

4. Rothman, L.S.; Gordon, I.E.; Barbe, A.; Benner, D.C.; Bernath, P.F.; Birk, M.; Boudon, V.; Brown, L.R.; Campargue, A.; Champion, J.-P.; et al. The HITRAN 2008 molecular spectroscopic database. J. Quant. Spectrosc. Radiat. Transf. 2009, 110, 533-572. [CrossRef]

5. Kaiser, J. The other global pollutant: Nitrogen proves tough to curb. Science 2001, 294, 1268-1296. [CrossRef] [PubMed]

6. Filho, M.B.; da Silva, M.G.; Sthel, M.S.; Schramm, D.U.; Vargas, H.; Miklós, A.; Hess, P. Ammonia detection by using quantum-cascade laser photoacoustic spectroscopy. Appl. Opt. 2006, 45, 4966-4971. [CrossRef] [PubMed]

7. Lundsbergnielsen, L.; Hegelund, F.; Nicolaisen, F.M. Analysis of the high-resolution spectrum of ammonia $\left({ }^{14} \mathrm{NH}_{3}\right)$ in the near-infrared region 6400-6900 $\mathrm{cm}^{-1}$. J. Mol. Spectrosc. 1993, 162, 230-245. [CrossRef]

8. Berden, G.; Peeters, R.; Meijer, G. Cavity-enhanced absorption spectroscopy of the $1.5 \mu \mathrm{m}$ band system of jet-cooled ammonia. Chem. Phys. Lett. 1999, 307, 131-138. [CrossRef]

9. Webber, M.E.; Baer, D.S.; Hanson, R.K. Ammonia monitoring near $1.5 \mu \mathrm{m}$ with diode-laser absorption sensors. Appl. Opt. 2001, 40, 2031-2042. [CrossRef]

10. Czajkowski, A.; Alcock, A.J.; Bernard, J.E.; Madej, A.A.; Corrigan, M.; Chepurov, S. Studies of saturated absorption and measurements of optical frequency for lines in the $v_{1}+v_{3}$ and $v_{1}+2 v_{4}$ bands of ammonia at $1.5 \mu \mathrm{m}$. Opt. Express 2009, 17, 9258-9269. [CrossRef]

11. Guelachvili, G.; Abdullah, A.H.; Tu, N.; Rao, K.N.; Urban, S.; Papousek, D. Analysis of high-resolution fourier transform spectra of ${ }^{14} \mathrm{NH}_{3}$ at $3.0 \mu \mathrm{m}$. J. Mol. Spectrosc. 1989, 133, 345-364. [CrossRef]

12. Martin, N.A.; Ferracci, V.; Cassidy, N.; Hoffnagle, J.A. The application of a cavity ring-down spectrometer to measurements of ambient ammonia using traceable primary standard gas mixtures. Appl. Phys. B 2016, 122, 219. [CrossRef]

13. Schilt, S.; Thevenaz, L.; Nikles, M.; Emmenegger, L.; Huglin, C. Ammonia monitoring at trace level using photoacoustic spectroscopy in industrial and environmental applications. Spectrochim. Acta Part A 2004, 60, 3259-3268. [CrossRef]

14. Manne, J.; Sukhorukov, O.; Jäger, W.; Tulip, J. Pulsed quantum cascade laser-based cavity ring-down spectroscopy for ammonia detection in breath. Appl. Opt. 2006, 45, 9230-9237. [CrossRef] [PubMed]

15. Shadman, S.; Rose, C.; Yalin, A.P. Open-path cavity ring-down spectroscopy sensor for atmospheric ammonia. Appl. Phys. B 2016, 122, 194. [CrossRef]

16. von Bobrutzki, K.; Braban, C.F.; Famulari, D.; Jones, S.K.; Blackall, T.; Smith, T.E.L.; Blom, M.; Coe, H.; Gallagher, M.; Ghalaieny, M.; et al. Field inter-comparison of eleven atmospheric ammonia measurement techniques. Atmos. Meas. Tech. 2010, 3, 91-112. [CrossRef]

17. Miller, D.J.; Sun, K.; Tao, L.; Khan, M.A.; Zondlo, M.A. Open-path, quantum cascade-laser-based sensor for high-resolution atmospheric ammonia measurements. Atmos. Meas. Tech. 2014, 7, 81-93. [CrossRef]

18. Maithani, S.; Mandal, S.; Maity, A.; Pal, M.; Pradhan, M. High-resolution spectral analysis of ammonia near $6.2 \mu \mathrm{m}$ using a cw EC-QCL coupled with cavity ring-down spectroscopy. Analyst 2018, 143, 2109-2114. [CrossRef]

19. Cottaz, C.; Kleiner, I.; Tarrago, G.; Brown, L.R.; Margolis, J.S.; Poynter, R.L.; Pickett, H.M.; Fouchet, T.; Drossart, P.; Lellouch, E. Line positions and intensities in the $2 v_{2} / v_{4}$ vibrational system of ${ }^{14} \mathrm{NH}_{3}$ near 5-7 $\mu \mathrm{m}$. J. Mol. Spectrosc. 2000, 203, 285-309. [CrossRef]

20. Owen, K.; Es-sebbar, E.; Farooq, A. Measurements of $\mathrm{NH}_{3}$ line strengths and collisional broadening coefficients in $\mathrm{N}_{2}, \mathrm{O}_{2}, \mathrm{CO}_{2}$, and $\mathrm{H}_{2} \mathrm{O}$ near $1103.46 \mathrm{~cm}^{-1}$. J. Quant. Spectrosc. Rad. Transf. 2013, 121, 56-68. [CrossRef] 
21. Abramov, P.I.; Kuznetsov, E.V.; Skvortsov, L.A.; Skvortsova, M.I. Quantum-cascade lasers in medicine and biology (review). J. Appl. Spectrosc. 2019, 86, 1-16. [CrossRef]

22. Elkan, R.B.; Mellors, R.C. Infrared spectra of tissues. Science 1949, 110, 137-138.

23. Bakalov, D.; Stoilov, M. Toward the measurement of the hyperfine structure of muonic hydrogen in the FAMU experiment. In Proceedings of the International Conference on Exotic Atoms and Related Topics—EXA2017, Vienna, Austria, 11-15 September 2017; p. 01033.

24. Adamczak, A.; Baccolo, G.; Bakalov, D.; Baldazzi, G.; Bertoni, R.; Bonesini, M.; Bonvicini, V.; Campana, R.; Carbone, R.; Cervi, T.; et al. Steps towards the hyperfine splitting measurement of the muonic hydrogen ground state: Pulsed muon beam and detection system characterization. J. Instrum. 2016, 11, P05007. [CrossRef]

25. Pizzolotto, C.; Adamczak, A.; Bakalov, D.; Baldazzi, G.; Baruzzo, M.; Benocci, R.; Bertoni, R.; Bonesini, M.; Bonvicini, V.; Cabrera, H.; et al. The FAMU experiment: Muonic hydrogen high precision spectroscopy studies. Eur. Phys. J. A 2020, 56, 185. [CrossRef]

26. Werle, P.; Mücke, R.; Slemr, F. The limits of signal averaging in atmospheric trace-gas monitoring by tunable diode-laser absorption spectroscopy (TDLAS). Appl. Phys. B 1993, 57, 131-139. [CrossRef]

27. Berden, G.; Peeters, R.; Meijer, G. Cavity ring-down spectroscopy: Experimental schemes and applications. Int. Rev. Phys. Chem. 2000, 19, 565-607. [CrossRef]

28. Jahjah, M.; Vicet, A.; Rouillard, Y. A QEPAS based methane sensor with a $2.35 \mu \mathrm{m}$ antimonide laser. Appl. Phys. B 2012, 106, 483-489. [CrossRef]

29. O'Keefe, A.; Deacon, D.A.G. Cavity ring-down optical spectrometer for absorption measurements using pulsed laser sources. Rev. Sci. Instrum. 1988, 59, 2544-2551. [CrossRef]

30. Romanini, D.; Lehmann, K.K. Ring-down cavity absorption spectroscopy of the very weak HCN overtone bands with six, seven, and eight stretching quanta. J. Chem. Phys. 1993, 99, 6287-6301. [CrossRef]

31. Zalicki, P.; Zare, R.N. Cavity ring-down spectroscopy for quantitative absorption measurements. J. Chem. Phys. 1995, 102, 2708-2717. [CrossRef]

32. Jongma, R.T.; Boogaarts, M.G.H.; Holleman, I.; Meijer, G. Trace gas detection with cavity ring down spectroscopy. Rev. Sci. Instrum. 1995, 66, 2821-2828. [CrossRef]

33. Ayers, J.D.; Apodaca, R.L.; Simpson, W.R.; Baer, D.S. Off-axis cavity ringdown spectroscopy: Application to atmospheric nitrate radical detection. Appl. Opt. 2005, 44, 7239-7242. [CrossRef]

34. Karpf, A.; Qiao, Y.; Rao, G.N. Ultrasensitive, real-time trace gas detection using a high-power, multimode diode laser and cavity ringdown spectroscopy. Appl. Opt. 2016, 55, 4497-4503. [CrossRef]

35. Faist, J.; Capasso, F.; Sivco, D.L.; Sirtori, C.; Hutchinson, A.L.; Cho, A.Y. Quantum cascade laser. Science 1994, 264, 553-556. [CrossRef] [PubMed]

36. Joly, L.; Zéninari, V.; Parvitte, B.; Weidmann, D.; Courtois, D.; Bonetti, Y.C.; Aellen, T.; Beck, M.; Faist, J.; Hofstetter, D. Spectroscopic study of the $v_{1}$ band of $\mathrm{SO}_{2}$ using a continuous-wave DFB QCL at $9.1 \mu \mathrm{m}$. Appl. Phys. B 2003, 77, 703-706. [CrossRef]

37. Curl, R.F.; Capasso, F.; Gmachl, C.; Kosterev, A.A.; McManus, B.; Lewicki, R.; Pusharsky, M.; Wysocki, G.; Tittel, F.K. Quantum cascade lasers in chemical physics. Chem. Phys. Lett. 2010, 487, 1-18. [CrossRef]

38. Yao, Y.; Hoffman, A.J.; Gmachl, C.F. Mid-infrared quantum cascade lasers. Nat. Photonics 2012, 6, 432-439. [CrossRef]

39. Myers, T.L.; Cannon, B.D.; Taubman, M.S.; Bernacki, B.E. Performance and reliability of quantum cascade lasers. In Proceedings of the SPIE Defense, Security, and Sensing, Baltimore, MA, USA, 29 April-3 May 2013; p. $87330 \mathrm{E}$.

(C) 2020 by the authors. Licensee MDPI, Basel, Switzerland. This article is an open access article distributed under the terms and conditions of the Creative Commons Attribution (CC BY) license (http://creativecommons.org/licenses/by/4.0/). 